\section{Cahiers de Narratologie}

Analyse et théorie narratives

$16 \mid 2009$

Images et récits

\title{
Dante n'est pas uniquement sévère (Dante no es únicamente severo) ou comment ne pas raconter une histoire
}

Jean-Paul Aubert

\section{OpenEdition}

Journals

Édition électronique

URL : http://journals.openedition.org/narratologie/1056

DOI : 10.4000/narratologie.1056

ISSN : 1765-307X

Éditeur

LIRCES

Référence électronique

Jean-Paul Aubert, «Dante n'est pas uniquement sévère (Dante no es únicamente severo) ou comment ne pas raconter une histoire », Cahiers de Narratologie [En ligne], 16 | 2009, mis en ligne le 05 janvier 2011, consulté le 30 avril 2019. URL : http://journals.openedition.org/narratologie/1056 ; DOI : 10.4000/ narratologie. 1056

Ce document a été généré automatiquement le 30 avril 2019.

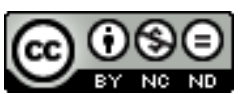

Cahiers de Narratologie - Analyse et théorie narratives est mis à disposition selon les termes de la licence Creative Commons Attribution - Pas d'Utilisation Commerciale - Pas de Modification 4.0 International. 


\section{Dante n'est pas uniquement sévère ( Dante no es únicamente severo) ou comment ne pas raconter une histoire}

Jean-Paul Aubert

1 Dante n'est pas uniquement sévère (Dante no es únicamente severo) est le titre énigmatique (emprunté à un roman d'Ilya Ehrenburg'1) d'une œuvre de cinéma réalisée en 1967 par deux cinéastes catalans, Joaquim Jordà et Jacinto Esteva. En sa qualité revendiquée de « vitrine » d'un mouvement cinématographique que l'on a appelé l'Ecole de Barcelone, Dante n'est pas uniquement sévère en synthétise les préoccupations et les choix esthétiques. Il est notamment emblématique d'un questionnement du récit et d'une réflexion sur la mise en œuvre de nouvelles formes narratives qui sont au cœur du chantier ouvert par ce mouvement et, plus largement, par les cinémas de la modernité dont il s'inspire et se réclame.

2 On rappellera en quelques mots la brève histoire de ce courant cinématographique à vocation avant-gardiste et expérimentale qui apparaît en Catalogne au milieu des années soixante. Disons d'emblée que le terme d'école peut sembler impropre pour désigner un mouvement qui ne prétend pas imposer un nouvel académisme pas plus qu'il n'entend perpétuer un modèle. L'Ecole de Barcelone réunit en un groupe informel de jeunes cinéastes qui, sur un plan politique, vouent une haine tenace au régime franquiste et qui, du point de vue de la création artistique, ont la volonté de s'émanciper des contraintes aussi bien administratives qu'esthétiques qui pèsent sur la création cinématographique en Espagne. Parmi les membres de ce mouvement, on compte un certain nombre de personnalités qui parviendront à se faire un nom dans le domaine du cinéma, comme réalisateurs, scénaristes ou critiques ou dans d'autres domaines, comme l'architecture. On peut citer pêle-mêle, outre Joaquim Jordà et Jacinto Esteva, Vicente Aranda, Román Gubern, José María Nunes, Pere Portabella ou encore Ricardo Bofill. L'Ecole de Barcelone s'éteint au début des années soixante-dix au terme d'une demi-douzaine d'années 
d'activité. Elle laisse à la postérité une vingtaine d'œuvres (longs et courts-métrages réunis) souvent déconcertantes et parfois hermétiques.

3 A l'instar de la plupart des mouvements qui se réclament de l'avant-garde, l'Ecole de Barcelone se place sous le signe du refus. Refus, évidemment, d'un cinéma complaisant, académique et routinier: des films historiques, des adaptations littéraires, des mélodrames, des comédies musicales, qui rivalisent de bondieuseries et de moralisme et déversent sur les écrans les images en carton-pâte d'une Espagne pittoresque et folklorique. Mais refus également d'un cinéma social et réaliste qui connaît un renouveau dans le cadre de ce que l'on a appelé le Nouveau Cinéma Espagnol. Ce courant qui naît à Madrid autour de l'Ecole Officielle du Cinéma est impulsé par les autorités franquistes elles-mêmes qui, dans une période où elles entendent donner des gages de modernité et d'ouverture, notamment vis-à-vis de l'étranger, accueillent très favorablement la perspective d'une Nouvelle Vague à l'Espagnole. Mais il est, concrètement et paradoxalement, le fait d'une nouvelle génération de réalisateurs qui, pour la plupart, ne cachent pas leur hostilité au régime et qui entendent s'engouffrer dans ce qu'ils croient être une brèche. Les premières œuvres que l'on associe au Nouveau Cinéma Espagnol, des films comme La Tía Tula (La Tante Tula, 1964) de Miguel Picazo ou Nueve cartas a Berta (Neuf lettres à Berta, 1965) de Basilio Martín Patino, osent pointer les contraintes idéologiques et morales qui pèsent sur la société espagnole. Mais le Nouveau Cinéma Espagnol se heurte très vite aux limites fixées par le régime, les cinéastes madrilènes se trouvant alors placés devant une alternative difficilement acceptable: soit composer avec la censure, soit renoncer purement et simplement à la réalisation de films susceptibles de lui déplaire. L'Ecole de Barcelone refuse de se laisser enfermer dans ce qui est, à ses yeux, une impasse. Constatant l'impossibilité dans une société de censure de mettre en oeuvre un cinéma chargé d'intention sociale, elle entend se tourner vers de nouvelles formes d'expression. "Etant donné que nous ne pouvons pas être Victor Hugo, nous serons Mallarmé.» Souvent citée, cette phrase prononcée par l'un des principaux animateurs de l'Ecole de Barcelone, Joaquim Jordà, peut donner la sensation d'un renoncement ou d'un choix par défaut. Mais, en invoquant l'héritage de Mallarmé, Jordà et, avec lui, L'Ecole de Barcelone, se placent surtout dans la perspective d'une révolution du langage poétique, la seule révolution qui dans une Espagne «ficelée et bien ficelée »-comme le dirait Franco luimême - leur semble désormais envisageable. Cette révolution passe par un refus des formes d'expressions et des conventions associées au réalisme et plus spécifiquement par une mise en cause du récit. Dante no es únicamente severo en offre une illustration radicale.

\section{Une genèse chaotique}

4 Pris dans son acception aristotélicienne le terme de « récit» désigne «l'agencement de faits en système ». Si l'on veut bien s'accorder sur cette définition consensuelle du récit comme processus d'assemblage et de mise en relation d'événements de sorte qu'ils se prolongent les uns dans les autres, on peut dire du film Dante no es unicamente severo qu'il n'en est pas un. Pour le dire simplement, Dante no es unicamente severo ne raconte rien. Mettant en œuvre une stratégie d'évitement du récit, ses auteurs se sont appliqués à disjoindre plutôt qu'à assembler, à délier plutôt qu'à relier.

5 La dispersion et l'éclatement s'invitent dans le film dès sa genèse. A l'origine de Dante no es unicamente severo, se trouve en effet le projet de réalisation de quatre courts métrages qui, une fois réunis, auraient constitué une sorte de manifeste cinématographique de 
l'Ecole de Barcelone. Pour l'occasion s'étaient associés deux maisons de production, Filmscontacto et Tibidabo Films et cinq réalisateurs : Antoni de Senillosa, Pere Portabella, Ricardo Bofill, Jacinto Esteva et Joaquim Jordà. Antoni de Senillosa projetait la réalisation d'un sketch intitulé Barcelona 66. Pere Portabella devait réaliser un court-métrage dont le titre, Carmen, lui avait été suggéré par Jordà qui s'en était remis au hasard de sa bibliothèque et était tombé sur le roman de Mérimée. Le titre du projet de Ricardo Bofill, Las cinco caras del cubo (Les cinq faces $d u$ cube), titre dans lequel on devine l'hommage au mouvement avant-gardiste héritier du dadaïsme, «Dau al set (la septième face du dé) » rend assez bien compte du dispositif scénique envisagé. Il consistait à placer trois personnages anonymes (deux femmes et un homme) dans un espace cubique, une sorte de boîte dont la sixième face serait occupée par la caméra. En réalité, il s'agit pour Bofill de poursuivre au cinéma la réflexion théorique engagée par le groupe du Taller de arquitectura dans le domaine de l'architecture autour du concept de "ville dans l'espace ». Ce concept qui sera formalisé en 1968 dans un ouvrage intitulé Hacia una formalización de la ciudad en el espacio envisage de développer l'espace urbain à partir d'une matrice, reproductible à l'échelle industrielle, le cube ${ }^{2}$. Le film offre donc au cinéastearchitecte l'opportunité d'observer, à la manière d'un entomologiste, l'évolution de personnages dans cette cellule type constituée par l'espace cubique. Le scénario original de Jacinto Esteva à pour titre La cenicienta. Una historia vertical (Cendrillon. Une histoire verticale) et propose une réinterprétation onirique et enfantine du conte d'Andersen. Enfin, Joaquín Jordà avait pour sa part rédigé un scénario intitulé provisoirement $+x-$ (más por menos), centré sur l'étrange histoire d'un homme poisson. Antoni de Senillosa se retirera très vite du projet. Les quatre épisodes restant devaient donc être réunis pour former un ensemble selon une pratique déjà expérimentée par la Nouvelle Vague française dans des films collectifs comme Les plus belles escroqueries du monde (1963) ou Paris vu par... (1965). Un document remis par Jacinto Esteva à la Commission chargée d'accorder une éventuelle subvention au projet définit en ces termes les ambitions de ce qui s'annonce comme le «manifeste » cinématographique de l'Ecole de Barcelone :

Volontairement éloigné de toute prétention réaliste, le film s'offre comme un produit résolument culturel, qui réunit les tendances les plus représentatives et avancées de l'art contemporain. Dans les quatre sketchs on trouve aussi bien le ballet abstrait, à la manière d'un Maurice Béjart -dans ofelia ${ }^{3}$ - que le graphisme publicitaire et la plastique moderne dans Cendrillon en passant par le film et le théâtre de l'absurde traités dans Carmen et Plus par moins ${ }^{4}$.

6 Les conflits entre les différentes parties auront finalement raison du projet initial tout en favorisant des combinaisons inattendues. Ricardo Bofill va se retirer assez rapidement du projet arguant de la nécessité de réaliser le film dans un format carré et en $35 \mathrm{~mm}$ incompatible avec les formats des autres films. Le scénario de Las cinco caras del cubo débouche donc sur un film intitulé d'une manière assez inattendue Circles. Carmen de Portabella devint un moyen-métrage intitulé No contéis con los dedos (Ne comptez pas sur vos doigts). Enfin, $+x-$ et La cenicienta. Una historia vertical vont être réunis en un seul film, Dante no es únicamente severo. Il y a, comme le voit, à l'origine des trois films un long processus d'associations et de dissociations qui témoigne en définitive d'une réelle confiance dans l'exploitation créatrice de l'alea. Le hasard des amitiés, des brouilles, des conflits d'intérêt devient un principe de création. Le résultat, ce sont ces trois films qui, tout en se voulant autonomes, poursuivent un même but : questionner le récit.

7 Dante no es únicamente severo, qui retiendra plus particulièrement notre attention, s'appuie, comme on l'a dit, sur deux scénarios originaux parfaitement indépendants dont 
le seul véritable point commun est la réflexion qu'ils engagent sur la narration. Selon Jordà :

Il s'agissait de deux histoires distinctes [...] dont le point de contact possible était l'idée d'un discours sur la narration, sur la possibilité de narration. [...] à cette époque, la question de savoir si la narration est terminée, si elle se poursuit, si elle n'existe pas, si l'on peut raconter, si l'on ne peut pas raconter, c'était très à la mode, et j'ai conçu une structure selon laquelle nous allions fractionner le matériel tourné de part et d'autre. On a inclus du matériel d'archive qui ne coûtait presque rien et l'on a tourné quatre ou cinq minutes de film et le résultat, ce fut Dante ${ }^{5}$.

Fidèle à ce principe, le film propose un agencement d'images et de sons dont on peine à déceler la cohésion et l'unité. Il faut dire que ses auteurs n'élaborent pas une synthèse des idées contenues dans les scénarios initiaux, mais se contentent de les assembler, ou plus exactement de les juxtaposer selon un collage que l'on pourrait juger à première vue désinvolte. Le prologue, fruit probable d'une improvisation, fut tourné par Esteva seul, hors de la présence de Jordà. On y voit des techniciens, des photographes, des topmodèles en discussion dans un parc dans l'attente probable d'un tournage. Suit un premier fragment, dû à Joaquín Jordà, qui comporte l'évocation d'un gigantesque incendie, la présentation d'une jeune top modèle interprétée par Serena Vergano et de son compagnon, joué par Enrique Irazoqui, et enfin l'illustration d'une nouvelle de Julio Cortázar. Un deuxième fragment fait alterner les images d'une opération de la cataracte, sur une idée de Jacinto Esteva, et des plans illustrant un conte animalier, L'anniversaire du lion, tournés par Jordà. Le troisième mouvement du film permet à Esteva de mettre en images sa vision démystificatrice du conte de Cendrillon. Les trois dernières parties, centrées sur le couple Serena Vergano/Enrique Irazoqui, sont de la responsabilité de Jordà. Le film s'achève sur un épilogue déconnecté en apparence de ce qui le précède immédiatement et qui est le seul fragment à avoir été réalisé en totale collaboration entre Jordà et Esteva. On y voit le transport vers un bloc opératoire de la jeune femme apparue dans le prologue. Dans une allusion très limpide à l'ouverture du chef d'œuvre du surréalisme Un Chien andalou, le film s'achève au moment précis où le chirurgien approche son bistouri de l'œil de la patiente. Cette dernière séquence est sensée, selon Jordà, assurer l'unité entre les différents éléments qui composent le film.

\section{L'hétérogénéité comme style, le hasard comme fil conducteur}

9 L'impression que l'on a affaire à un patchwork est renforcée par l'hétérogénéité de l'image et du son et par les ruptures constantes de registres et de tons. La couleur alterne avec le noir et blanc, les images en mouvement avec les photos fixes, le film de fiction avec des images documentaires ou des films d'actualités cinématographiques, la prise de son directe avec la post-synchronisation, les mélodies les plus suaves avec les musiques les plus tonitruantes. Les images et les sons de natures et de textures différentes se rencontrent, s'affrontent, se percutent. Le montage cultive la discontinuité et défie toute logique. Les plans, les séquences se succèdent dans une apparente incohérence. C'est une simple juxtaposition de choses sur le mode de l'inventaire. Aux images délicates d'un parc majestueux succèdent des vues apocalyptiques sur les ruines encore fumantes d'un bâtiment qui vient de brûler. Les images d'une course de Formule 1 s'intercalent entre celles d'un défilé de mode. Un bref film documentaire montrant des têtes de lions présentées comme des trophées est suivi d'images d'une jeune femme déambulant devant 
des affiches publicitaires, elles-mêmes précédant un plan large du Nou Camp, le stade de football de Barcelone. Et soudain ce bric-à-brac de sons et d'images s'interrompt pour faire place à des micro-récits parfaitement autonomes au sens où ils n'ont pas plus de liens entre eux qu'ils n'en ont avec ce qui les précède ou ce qui les suit. Ainsi au sein de cet ensemble très faiblement narratif qu'est le film, apparaissent, comme autant d'îlots, des récits dotés d'une relative cohérence narrative. C'est par exemple, sur le mode de la fable animalière, l'histoire d'un lion qui décide d'organiser une fête où tous les animaux sont invités sauf la grenouille. C'est encore, inspiré d'une nouvelle de Julio Cortázar, le récit d'un homme qui se rend quotidiennement au jardin zoologique pour admirer le ballet des poissons jusqu'à ce jour où, apercevant son reflet dans la vitre de l'aquarium, il connaît la révélation: ce qu'il voit, ce ne sont pas les poissons, c'est un homme qui observe des poissons. Ces récits interpolés introduisent des bifurcations soudaines dans une trame diégétique placée sous le sceau de la discontinuité. Ils creusent un espace de narration dans un ensemble dysnarratif, créent un désir d'unité et de continuité dans un contexte général où la disjonction et la discontinuité sont la règle.

Hormis ces instants de répit où le spectateur se voit offrir l'ébauche d'un récit linéaire et complet, le film demeure fidèle à un principe d'écriture fondé sur la déliaison. Ses auteurs semblent avoir mis un acharnement particulier à ruiner la logique de l'action qui gouverne la narration classique. La suite des événements n'obéit pas à une construction fondée sur une relation de cause à effet. Ce principe d'un montage cinématographique basé sur le refus de la logique fait l'objet d'une sorte de mise en abyme dans le dialogue suivant:

Lui : 1) Toute idée sur mon compte qui ne peut pas être exprimée sous forme d'un syllogisme est véritablement ridicule.

2) Aucune de mes idées sur les plum-cakes ne mérite d'être écrite.

3) Aucune des idées que je ne parviens pas à peindre ne peut être exprimée sous la forme d'un syllogisme.

4) Il n'y a pas d'idée véritablement ridicule dont je ne fasse part à mon avocat.

5) Je ne rêve que de plum-cakes.

6) Je ne fais part à mon avocat d'aucune idée qui ne mérite pas d'être écrite.

Conclusion?

Elle : Ce n'est pas très difficile : tous mes rêves se réalisent.

Lui : parfait 6 .

11 Le dialogue s'empare du syllogisme, modèle déductif par excellence, pour donner à l'absurdité toutes les apparences de la logique la plus rigoureuse. Mais au-delà de la facétie et du jeu avec les mots, l'écart entre les prémisses du syllogisme et sa conclusion improbable témoigne de l'impossibilité d'accéder à une connaissance déductive du réel. L'impossibilité d'un enchaînement obligatoire des arguments suivant la règle de la logique la plus élémentaire transcrit dans les dialogues une pratique du montage fondée sur la dislocation de l' "image-action", pour reprendre une expression chère à Gilles Deleuze. La recherche de liens sensori-moteurs dans une œuvre qui s'acharne à les détruire semble donc vouée à l'échec. Dépourvus des liens syntaxiques de la causalité, privés de connecteurs logiques, les plans et les séquences tirent leur valeur de leur dissociation et non d'une hypothétique association. 


\section{Les malheurs de Shéhérazade}

12 On peut comprendre le désarroi et même l'irritation du spectateur face à un film qui semble n'offrir aucune prise à l'analyse et pose effectivement le problème d'une limite de la réception. C'est une frustration que partagent certains critiques. Ainsi Juan Antonio Martínez Bretón conclut-il son analyse du film par ces quelques mots désemparés :

Le film est un casse-tête doté d'un engrenage obscur et compliqué. C'est le plus grand défaut que nous trouvons à Dante no es unicamente severo ${ }^{7}$.

13 Dans cette phrase perce le désappointement de celui qui s'est efforcé en vain de résoudre l'énigme proposée par le film. Faut-il conclure, comme le suggère cette remarque désabusée du critique, à un complet naufrage du sens?

Dante no es unicamente severo est un non-récit, voire un anti-récit qui fait de l'impossibilité de raconter son seul véritable thème. Celui-ci s'annonce dès les premiers instants du film. Peu après le prologue, deux cartons devancent l'apparition d'une jeune femme incarnée par Serena Vergano: "Au commencement était une femme» («Al principio era una mujer»); «et cette femme parlait» («y esa mujer hablaba»). Ces deux intertitres annoncent une omniprésence du verbal qui ne sera jamais démentie tout au long du film. On y découvre en effet un étonnant inventaire des possibilités qu'offre le matériau verbolinguistique aussi bien dans sa composante sonore (monologues, dialogues, bavardages, slogans commerciaux, récitation d'une fable, voix d'un commentateur) que dans sa composante écrite (cartons divers, textes théoriques inscrits sur l'écran, enseignes publicitaires). Mais une part prépondérante du discours va être pris en charge par la jeune femme qu'interprète Serena Vergano. Le spectateur ne tardera pas à découvrir les malheurs de cette Shéhérazade moderne qui s'évertue à raconter ses histoires à un homme qui les dédaigne. C'est en vain qu'elle essaie d'attirer l'attention de son interlocuteur masculin qui oppose à ses sollicitations la distance et le mépris. Cette communication déjà difficile est en outre constamment empêchée par l'intrusion intempestive d'éléments extérieurs qui ruinent les tentatives de la jeune femme de raconter une histoire. Elle n'y parviendra que de manière ponctuelle dans les quelques moments narratifs décrits plus haut. Une part essentielle du film se joue ainsi dans cette tension entre les îlots de continuité narrative que constituent ces micro-récits, et l'océan de discontinuité dans lequel le film semble en définitive s'engloutir. Tension entre fluidité et entraves, entre conjonction et disjonction.

Quels sont les éléments perturbateurs du récit? Nous en avons déjà cité quelques-uns : des vues d'un défilé de mode, des images d'un mannequin déambulant parmi les passants dans les rues de Barcelone, un reportage sur une course automobile, des spots publicitaires. Ce sont aussi des sons. L'espace sonore est en effet régulièrement parasité par des discours ou par les comptes-rendus officiels récités docilement par des journalistes de radio. On en a un exemple dans le deuxième mouvement du film. La scène se situe dans une luxueuse chambre à coucher tandis qu'une voix-off rend compte d'une soirée organisée par la Direction d'une entreprise :

Le jeudi 28 juillet un dîner a réuni dans un restaurant typique de Barcelone la quasitotalité des employés administratifs de l'entreprise, y compris nos camarades de l'usine de Sabadell. La soirée s'est déroulée dans le climat d'amitié et de camaraderie qui caractérise notre entreprise. A la table présidentielle était assis notre cher Directeur Général, Francisco Jordà, accompagné du conseiller délégué. A la fin du dîner offert par M. Jordà en hommage aux employés de l'entreprise, celui- 
ci a invité les participants à poursuivre leur travail remarquable au service de l'entreprise, une entreprise à laquelle ils doivent faire confiance en toute circonstance. avertissement qui semble s'adresser directement au spectateur imite le discours d'un pouvoir autoritaire :

Avertissement: la projection du film est suspendue jusqu'au complet rétablissement de l'ordre. Les autorités font confiance au bon sens et à la sagesse de la majorité de la population qui ne tarderont pas à prendre le dessus sur les intentions subversives et inavouables d'une minorité8.

Le récit voit ainsi sa cohérence et sa linéarité sans cesse remises en cause. Il semble qu'il est en proie à un parasitage constant, comme victime du «bruit de fond» d'une société qui ressemble à s'y méprendre à la société espagnole des années soixante. On y perçoit les vociférations des autorités mais aussi le bourdonnement des soirées mondaines et le ronronnement de l'information. Mais ce brouhaha bien connu cède le pas à un tumulte encore insolite pour l'époque, celui d'une société en passe de succomber à la fièvre consumériste et d'ores et déjà gagnée par la sophistication, l'artifice et la frivolité. C'est le monde de la mode, du luxe, de l'oisiveté qui s'invite dans les images récurrentes de top modèles prenant la pose ou dans des plans insistants sur des panneaux publicitaires.

Si le récit est condamné à l'inaboutissement, c'est que toute tentative pour raconter est désormais contrariée par ce déferlement des images et des sons que produit la société de consommation naissante et qui semble déjà inviter l'individu à ce que l'on appellera plus tard la pratique du zapping. En montrant de quelle façon l'espace public est envahi par ce «bruit de fond » de plus en plus tenace, le film se fait le témoin d'une société en profonde mutation, marquée notamment par l'apparition et le développement rapide de la télévision, l'essor de la publicité, la surinformation. Rappelons que la télévision d'Etat débute ses émissions en 1958, qu'en 1959, on compte déjà en Espagne 30000 postes de télévision. Dix ans plus tard, en 1969, 62 \% des foyers espagnols en sont pourvus. Il faut également garder à l'esprit la situation particulière de Barcelone et de la Catalogne, probablement la région la plus riche d'Espagne dans les années soixante, la première également à avoir fait de la publicité une industrie avec son armada de photographes, de modèles et de sociétés spécialisées. Ce n'est pas le moindre des paradoxes de Dante no es únicamente severo: ce film, qui récuse la tentation du réalisme, se livre finalement à un constat de type sociologique. Un constat partagé du reste par d'autres films de l'Ecole de Barcelone comme No contéis con los dedos de Pere Portabella qui repose sur une accumulation de spots publicitaires ou Fata Morgana de Vicente Aranda qui s'élabore autour de la figure d'un mannequin à succès dont l'image s'étale dans les magazines et sur les panneaux publicitaires.

19 Mais Dante no es únicamente severo va peut-être au-delà en envisageant les effets de ce déferlement d'images et des sons liées à la mode, au design ou à la publicité sur l'imaginaire des individus. Tout se passe comme si la caméra avait pu s'insinuer dans le cerveau du personnage masculin et y saisir les images qui s'y forment tandis que la jeune femme raconte ses histoires. L'hypothèse que nous faisons est que Dante no es únicamente severo se place du point de vue de la réception psychique du récit. C'est l'expérience sensorielle et imaginaire du récepteur que le film s'efforce de décrire. Autrement dit, les images qui défilent sur l'écran ne correspondent pas à l'histoire telle qu'elle est imaginée par l'énonciatrice mais à l'histoire telle qu'elle est perçue et interprétée par le récepteur. $\mathrm{Au}$ personnage féminin, le pouvoir de la parole ; à son partenaire masculin, celui de créer 
les images. Or, l'interprétation fruit de l'indétermination du texte est un exercice qui mêle la perception, l'effort de compréhension et l'expérience personnelle. Ainsi que le dit une voix-off impersonnelle au cours du film, si « une image peut conduire à une histoire, une histoire ne peut jamais conduire à une image mais à une multitude confuse d'images ". D'où le hiatus constant entre les paroles prononcées par la narratrice et les images engendrées par l'imaginaire du récepteur.

La multiplication des cartons et des intertitres met en lumière le caractère antinomique du verbal et de l'iconique. Le surgissement de l'un produit en effet la suspension de l'autre. L'apparition de cartons sur lesquels figurent des indications temporelles (dans l'esprit du cinéma muet) ou diverses sentences contribue à la fragmentation du monde diégétique figuré et participe de la discontinuité et de l'hétérogénéité constitutives du film. De l'antinomie à l'indépendance, il n'y a qu'un pas que le film franchit à plusieurs reprises, dès lors qu'il renonce à faire correspondre le visuel à l'énoncé verbal. Cette disjonction pour ne pas dire ce conflit entre le mot et l'image qu'il suggère est au cœur de plusieurs séquences. Par exemple le mot «un león» prononcé par la voix-off semble engendrer l'image d'un lion qui ne convient pas à l'énonciateur puisqu'il la récuse aussitôt en exigeant qu'apparaisse un autre lion. Plus loin dans le film, la réinterprétation mentale que le personnage masculin fait de la figure du prince charmant dans le conte Cendrillon est également significative d'une possible dichotomie entre le mot et l'image. A partir de l'expression « príncipe azul » qui fait qu'en espagnol le « prince charmant » est un « prince bleu », le personnage imagine un prince aux allures de vampires, vêtu de bleu, et les yeux injectés de sang. Le film fait ici l'expérience de l'autonomie de l'image sonore et de l'image visuelle et de l'impossibilité de prolonger simplement la première dans la seconde. La théorie que développeront André Gaudreault et François Jost selon laquelle le cinéma sonore propose un double récit, l'un porté par la voix et l'autre par l'image se voit ici démontrée par l'absurde ${ }^{10}$. Tandis que le cinéma de facture classique s'efforce d'articuler d'une manière cohérente les deux récits de manière à assurer la compréhension de la totalité de l'intrigue, Dante no es únicamente severo s'applique à dénouer les liens entre le verbe et l'image.

21 Le film de Jordà et Esteva renouvelle en la radicalisant une expérience qui sera abondamment mise en œuvre par le cinéma moderne. Les œuvres de Marguerite Duras, Straub ou Robbe-Grillet exploitent largement l'autonomie possible du son et de l'image. Dans l'œuvre de Duras, l'écart se creuse entre le visuel et le sonore depuis l'a-synchronie étudiée entre les mots prononcés et les mouvements des lèvres des personnages jusqu'à la totale autonomie des bandes son et images, scellée dans un film comme Son nom de Venise dans Calcutta désert (1976). Chez Robbe-Grillet, le visuel et le parlant se contredisent sans cesse. L'Homme qui ment (1968) joue de cette contradiction à plusieurs reprises. Par exemple la voix-off dit d'une auberge qu'elle est vide alors que l'image la montre pleine de monde. On aurait évidemment tort de croire que la coupure entre le sonore et le visuel débouche sur un congé du sens. C'est au contraire l'interstice qui sépare le sonore du visuel qui doit faire sens désormais, révélant l'instabilité du réel, l'indiscernabilité du vrai et du faux et la fin de la toute puissance de la parole. Sur ce dernier point en particulier, Dante no es únicamente severo reprend une fois de plus à son compte une découverte que l'on a souvent imputée aux cinémas de la modernité. On aura remarqué l'ironie des cartons qui précèdent l'apparition de la conteuse ( « Au commencement était une femme. Et cette femme parlait») qui font échos aux premiers mots de l'Evangile de Jean («Au commencement était le verbe »). Ainsi un film qui va s'employer à démontrer 
l'impuissance du verbe débute-t-il par l'évocation malicieuse d'un texte qui témoigne de la foi en la force créatrice de la parole. Ironie encore de la référence implicite à la parole salvatrice de Shéhérazade dans un film où les mots ne font que creuser le fossé d'indifférence entre celle qui les prononce et celui qui les écoute. Mais, à la différence d'un film comme L'Homme qui ment où le sonore et le visuel prennent en charge alternativement le réel et l'imaginaire, dans Dante no es únicamente severo, ils se chargent l'un et l'autre d'imaginaire. Le hiatus entre le sonore et le visuel rend compte de l'impossible rencontre entre l'imaginaire de la fabulatrice à l'origine des mots et l'imaginaire du récepteur de la fable à l'origine des images. La parole se perd, inapte à faire le lien entre deux imaginaires. Cet intérêt de Jordà pour comprendre les ressorts de la perception trouvera dans son œuvre un prolongement inattendu. Peu de temps avant sa mort, Jordà réalise un documentaire intitulé Más allá del espejo (Au-delà du miroir). C'est une plongée dans le monde de l'agnosie et de l'alexie, des maladies cérébrales qui altèrent la perception de ce que l'on appelle communément la réalité. L'agnosie, qui peut être visuelle, auditive ou tactile empêche de reconnaître les objets. L'alexie est une incapacité à reconnaitre les éléments du langage. On parle fréquemment dans ce cas de "cécité verbale ». Souffrant lui-même de cette affection consécutive à un accident vasculaire cérébral, Jordà part à la rencontre d'autres personnes atteint de ce mal étrange. L'ironie $\mathrm{du}$ sort conduit ainsi le réalisateur à poursuivre dans sa dernière œuvre une réflexion qu'il entamait dans son premier film. Más allá del espejo avance des explications cliniques pour tenter d'expliquer les difficultés à appréhender le monde. Dans Dante no es únicamente severo l'alexie dont semble souffrir le personnage masculin a des causes d'ordre aussi bien psychiques que sociologiques : les images et les sons qui naissent dans le cerveau du récepteur en réponse à la parole créatrice de la fabulatrice apparaissent comme une représentation des pensées obsessionnelles, des rêves ou des fantasmes dont le récepteur ne peut se défaire et qui interfèrent avec les images suggérées par la narration.

La déconstruction du récit est probablement l'une des données majeures de la modernité. Elle va trouver en l'Ecole de Barcelone des adeptes inventifs d'autant plus appliqués à en investir chacune des modalités qu'ils ont acquis la conviction que la contestation du récit conventionnel est l'unique licence que leur accorde le régime franquiste. Dante no es únicamente severo est, de ce point de vue, une œuvre exemplaire où se concrétise l'ambition énoncée par Joaquín Jordà lors du Festival de Pesaro : "Aujourd'hui, il n'est pas possible de parler librement de la réalité de l'Espagne, nous tentons donc de décrire son imaginaire ». Et, pour ses auteurs, l'imaginaire des Espagnols apparaît aussi sinistré que leur réalité. Submergé, vampirisé ou peut-être même tari par le flot ininterrompu des images et des sons que produit la société bourgeoise, l'imaginaire est devenu inapte à produire et à accueillir du récit. L'impossibilité du récit est une donnée de l'époque, de cette époque nouvelle qui s'ouvre en Espagne dans les années soixante. Le film s'évertue à ne recueillir du récit que des bribes éparses, des tentatives avortées, une succession de ratages. En échos à cet inaboutissement constant de la narration et aux échecs répétés de Shéhérazade, le spectacle offert par le personnage masculin, vautré sur un lit ou absorbé dans la réalisation de cocottes en papier est celui d'une misère intérieure, d'un dessèchement de l'esprit, reflet de la superficialité et de la vacuité dans laquelle se complaît la société bourgeoise. Le vide s'installe. Il est inhérent à la discontinuité du récit, il s'insinue dans ses failles et dans ses ratés, en écho à une déchirure qui affecte les êtres, reclus dans leur solitude, lacérés intérieurement. Après avoir gagné le discours des personnages le faisant sombrer dans la banalité et la futilité, il s'empare de l'espace. La 
dernière partie du film se déroule au sommet d'un immeuble en chantier, dans un appartement sans porte, sans fenêtre ni cloison, ouvert sur le vide.

Quel avenir pour ce monde sans finalité, qui confine au néant? La destruction. «Cela suffit, finalement la destruction est préférable au néant » s'exclame la jeune femme dans l'une des dernières séquences. Cette hypothèse de l'anéantissement a été instillée tout au long du film. C'est ainsi qu'il semble que chaque chose peut s'annuler en son contraire. Les premiers instants de Dante... invitent le spectateur à découvrir un jardin édénique. Au détour d'une allée ombragée, une Eve à la blondeur angélique laisse admirer sa plastique parfaite sous des voilages d'un bleu transparent. Mais, le spectateur est brutalement détourné de ce rêve d'un paradis originel par les images apocalyptiques d'un gigantesque incendie, tandis que la délicieuse musique fait place aux bruyantes et terrifiantes acclamations d'un rassemblement nazi. Les images d'incendie sont réitérées d'une manière quasi obsessionnelle tout au long d'un film qui s'achève, comme il avait presque commencé, sur des images de destruction. Au terme d'un compte à rebours, le fracas d'une explosion de grande ampleur se fait entendre tandis que l'écran prend des couleurs rougeâtres virant au jaunâtre puis au vert. Le film qui s'était inspiré plus tôt des premiers mots de l'Evangile de Jean s'achève ainsi sur une évocation de l'Apocalypse s'offrant comme un raccourci du destin de l'humanité et ne lui offrant d'autre issue que sa Chute dans un enfer qui pourrait ressembler à celui de Dante.

\section{NOTES}

1. Esteve Riambau et Casimiro Torreiro, La Escuela de Barcelona: le cine de la "gauche divine", Anagrama, Barcelone, 1999, p. 161.

2. Ricardo Bofill, Taller de Arquitectura, Hacia una formalización de la ciudad en el espacio, Barcelone, Blume, 1968.

3. Ofelia est le sous-titre de l'un des scénarios du film de Ricardo Bofill.

4. Cité par Esteve Riambau et Casimiro Torreiro, La Escuela de Barcelona: le cine de la "gauche divine ", op. cit., p. 165.

5. Juan Antonio Martínez Bretón, La Denominada "Escuela de Barcelona», Editorial de la Universidad Complutense, Madrid, 1984. pp. 100-101.

6. «El: $1^{\circ}$ - Toda idea sobre mí que no pueda expresarse como forma de silogismo es verdaderamente ridícula.

2- Ninguna de mis ideas sobre los plum-cakes merece ser escrita.

3- Ninguan de las ideas que no llego a pintar, puede ser expresada bajo silogismos.

4- Nunca tengo una idea verdaderamente ridícula, sin consultarla inmediatamente con mi abogado

5- Sólo sueño con plum-cakes.

6- No consulto ninguna idea con mi abogado, a menos que no valga la pena escribirla.

¿Conclusión?

Ella : No es tan difícil : todos mis sueños se realizan.

$\mathrm{El}$ : perfecto. 
7. «El film es un rompecabezas provisto de un engranaje oscuro y complicado. Este es el mayor defecto que encontramos en Dante no es únicamente severo. » Juan Antonio Martínez Bretón, La Denominada « Escuela de Barcelona », op.cit., p. 136.

8. «Aviso : se suspende la proyección de la película hasta el total restablecimiento del orden. La autoridad confía en que el buen sentido y la sensatez de la mayoría no tardarán en imponerse sobre las intenciones subversivas e inconfesables de unos pocos ».

9. «Una imagen puede conducir a una historia. Una historia jamás a una imagen sino a una multitud confusa de imágenes. »

10. André Gaudreault, François Jost, Le Récit cinématographique, Nathan, coll. «Fac cinéma », Paris, 1990, p. 28-30.

\section{RÉSUMÉS}

Dante no es únicamente severo [Dante n'est pas uniquement sévère], un film de Joaquín Jordà et Jacinto Esteva, s'est voulu une vitrine de l'Ecole de Barcelone. Ce courant cinématographique actif à partir de 1965 et jusqu'au début des années soixante-dix, s'est notamment distingué par son refus de l'orthodoxie du récit. A ce titre, il a pu apparaître comme un avatar des cinémas de la modernité. Mais, l'Ecole de Barcelone et ce film qui en synthétise les principes doivent être envisagés dans le contexte particulier de l'Espagne sous le franquisme. La contestation du récit y acquiert une dimension politique et éthique.

Dante no es únicamente severo, una película de Joaquín Jordà y Jacinto Esteva, pretende hacer la síntesis del proyecto estético de la Escuela de Barcelona (una corriente cinematográfica activa desde del año 1965 y hasta principios de la década de los setenta). Por su rechazo de la ortodoxia del relato la Escuela de Barcelona se sitúa en la continuidad de los cines de la modernidad. Sin embargo, conviene examinar esta corriente y este film, en particular, en el contexto particular de la España franquista, donde el rechazo del relato abarca dimensiones política y éticas.

\section{INDEX}

Index chronologique : XXe siècle

Index géographique : Espagne

Mots-clés : Jordà Joaquín, Esteva Jacinto, Ecole de Barcelone, récit

\section{AUTEUR}

JEAN-PAUL AUBERT

Université de Nice-Sophia Antipolis, CIRCPLES EA 3159 